\title{
WAY TO SMART LIFE USING DATA MINING
}

\author{
Mrs. P. Kavya Nikeeta ${ }^{1}$ \\ Assistant Professor, Department of Computer Science Engineering, \\ Raghu Institute of Technology, Visakhapatnam, India. \\ Mr. Ch. Venkateswara Rao ${ }^{2}$ \\ Assistant Professor, Department of Information Technology, \\ Gudlavalleru College of Engineering, Gudlavaller, India.
}

Article DOI: $\underline{\text { https://doi.org/10.36713/epra6031 }}$

\begin{abstract}
Information offers numerous prerequisites to the end clients, for example, programming, association and stage go on. In this proposed framework, we concentrate about carefully mining the information on social media. Online media turns out to be well known from the information given by biomedical and other providers. This data is usually shared so that medical service improves, costs declines and utilises the assessment which is created by client. We suggest investigation framework that give attentions on side effects of drugs and also focus on positive and negative response. To improve health care some Clinical documents are mostly useful because these are free text data sources. Clinical reports containing data identified with manifestations and significant meds. Extracting data from enormous dataset became famous in light of the fact that clients get different thoughts from this shifted information. Data Mining and Knowledge mining became well known on the grounds that clients are aware of information and getting data of various region like wellbeing, Social, etc. After information preparing we center on clients positive and negative assessments. We exclude these feelings and discover which prescription is great; to choose this we additionally discover the symptoms of the drugs. Further we center on the manifestations of the sickness of tolerant. By taking the master specialists proposal, we rattle off the medicine of any sickness as per the side effects and we give this medicine or treatment to the client on our gathering. We can grow our examination into Data and Knowledge mining of online media and takes the client's reviews on different medications of Illness. This day by day refreshed information serves to drug industry, specialists, emergency clinics, and clinical staff, for viable future medicines.
\end{abstract}

KEYWORDS: Information mining, Complex organizations, social figuring, Data mining, semantic Web. 


\section{INTRODUCTION}

Web-based media is giving endless occasions to patients to offer their input about specific medications and gadgets, numerous associations can likewise obtain criticism on their administrations and items. Online media is giving unlimited events to patients to offer their contribution about explicit drugs and contraptions, various affiliations can similarly acquire analysis on their organizations and things. It offers to decorate and upgrade dispatching. This advancement serves to decline charges and lastly it builds benefit and turnover. Realities of Social media are gathering for bio examination moreover. Web-based media licenses a VNE(virtual systems administration climate) We can remove Knowledge by utilizing different computational devices. By utilizing accessible networks making a gathering of online media is likewise one of the method of information separating. . By utilizing accessible networks making a gathering of webbased media is likewise one of the method of information removing. Informal community is much the same as a structure which is an assortment of edges and hubs. These hubs, edges are associated with one another in various connections.

Because of the quick improvement of advanced literary content, insights made to be had in most recent years, text Mining, Data mining, measurement mining constant of information turns out to be more famous to change over such data in to helpful records and important information. Information mining can be considered as the extraction of crude or futile information from immense information bases, numerous applications like medical care frameworks, market investigation get focal points by such mined information and furthermore they came to realize how to remove valuable information from a major measure of information. This extracted information is generally helpful to client. Clients Internet battling came to be totally well known with net visit the utilization of social sites. Presently people will give explicit scrutinizes on net site on the web, similar to a monstrous U. S. India scope of individuals are utilizing social sites to tweet, talk. So this web records become an absolutely basic component to people groups who need to get barely any quite negative measurements of own personal field. Very Significant Data are dispatched with conversation board technique we have totally loved data identified with wellness care since all topic going related with most Disease furthermore, related welcomed on and medication. So we need mindfulness in these important realities as resources. These strategies are to be needed to get data and framework on realities yet this data having some restriction because of the reality this is capable to compositions on example model least complex strategy we just procedure on limited quantity of insights and get a little example of realities.

\section{STATEMENT OF PROBLEM}

The primary goal is to propose a novel way to deal with object compromise that depends on a current semantic similitude measure for connected information. We adjust the measure to the item compromise issue, present careful and surmised calculations that productively execute the strategies, and give a precise exploratory assessment dependent on a benchmark dataset.

This methodology can extend examination into shrewdly digging web-based media information for shopper assessment of different therapies to give fast, exceptional data for the drug business, emergency clinics, and clinical staff, on the viability (or ineffectualness) of future medicines.

\section{REVIEW OF LITERATURE}

An easy way to comply with the conference paper formatting requirements is to use this document as a template and simply type your text into it. Many people's are works on the cancer treatments to improve health care.

Si Yan and Yanliang Qi worked on cancer research they used the 3 various text mining tools namely MedLEE, HITEx and caTIES. These tools are developed by Columbia University and Harvard University these tools helps to extract medical information and diagnosis from pathology report. After extracting information they made one hypothesis which contains the specific type of cancer and drugs used for same.

Jun Huan, Wei Wang, Jan Prins algorithm FFSM for the frequent subgraph mining problem. Comparing to existing algorithms, FFSM achieves substantial performance gain by efficiently handling the underlying sub graph isomorphism problem.

Alberto Ochoa , Arturo Hernndez shows study about artificial Societies and Social Simulation using Ant Colony, Particle Swarm Optimization and Cultural Algorithms.

Jan Noessner, Mathias Niepert, Christian Meilicke, and Heiner Stuckenschmidt . In this paper, we propose a novel approach to object reconciliation that is based on an existing semantic similarity measure for linked data. We adapt the measure to the object reconciliation problem, present exact and approximate algorithms that efficiently implement the methods, and provide a systematic experimental evaluation based on a benchmark dataset.

Mr. Pramod B. Deshmukh , Mrs. Aditi A. Kalia Mrs. Vrushali U. Utterwar , Mrs. Dipali M. Patil shows study on Intelligently extracting knowledge from social media has newly attracted great interest from the Biomedical and Health Informatics community to simultaneously improve healthcare result and moderate costs using consumergenerated viewpoint that is from opinion mining. 
T. Anisha , Mr. N. Thulasi This approach can expand research into intelligently mining social media data for consumer opinion of various treatments to provide rapid, upto-date information for the pharmaceutical industry, hospitals, and medical staff, on the effectiveness (or ineffectiveness) of future treatments.

S. Arul Kiruba, V. Pavithra, A. Saranya and B. Dharani. To improve the care of human health by consumers opinion from the forum posts is our aim. he propose a system for discovering and extracting a positive and negative symptoms and side effects of different drugs for lung cancer disease from influential users forum posts. Based on these outcomes the drugs are rated and ranked based on TF-IDF.

Sonali More, P. P. Joshi Survey on Social Media Data Mining Techniques for detecting useful knowledge from massive datasets like trends, patterns and rules. This survey discusses different data mining techniques used in mining social media.

\section{DRAWBACK OF EXISTING SYSTEM}

1. Mining Structural Unstructured Data is convoluted.

2. In Existing framework Lack of information in Social organization examination.

3. Existing framework Implemented uniquely for malignancy infection.

4. Existing framework can't transfers remedy paper for quiet.

\section{PROPOSED SYSTEM}

Online media is introducing endless open doors for patients to offer their input about specific medications and gadgets, numerous associations can likewise get input on their ser-indecencies furthermore, items. Clinical associations are giving first need to interpersonal organization following in their Information Innovation offices, making opportunities for fast conveyance and items related remarks. It offers to improve also, enhance dispatching. This enhancement assists with diminishing charges lastly it expands benefit and turnover. Realities of Web-based media are gathering for bio Investigation additionally referenced also. Online media grants a VNE (virtual networking climate). We can separate Knowledge by utilizing different computational apparatuses. By utilizing accessible organizations making a gathering of online media is likewise a one of the method of information extricating. Informal community is much the same as a structure which is a assortment of edges and hubs these hubs and edges are associated with one another in various connections. Due to the quick increment and improvement of computerized literary substance measurements made to be had in most recent few years, text Mining, Data mining, measurement mining constantly of information becomes more famous to change over such data in to advantageous records and important information. Information mining can be considered as the extraction of crude or pointless information from enormous information bases, numerous applications like medical services frameworks, market examination get focal points by such mined information and furthermore they came to know how to extricate valuable information from a major measure of information. This removed information is generally valuable to client. Clients Internet battling is come to be a totally popular with net visit the utilization of a social sites. Presently people will give explicit evaluates on net site on the web, similar to a monstrous U. S. India range of individuals are utilizing social sites to tweet, visit. So this web records become an absolutely basic component to people groups who need to get a couple of overall quite negative insights of own field. Numerous strategies had been working like comprising of connection mining, type by means of hyperlinks, forecasts essentially based on things hyperlinks, ways of life, assessment, object, establishment, and subgroup discovery, and information mining. By utilizing people criticism Link forecast, biochemical promoting, online conversation organizations (and rankings) grant for improvement of answers. Important Data are dispatch with conversation board technique we have a totally cherished data identified with wellness care since all topic going related with most malignancies and related welcomed on also, medication. So we need to mindfulness in these significant realities as resources. This all techniques are to be needed to get data and framework on realities yet this data having some constraint due to the reality this all ready to works of art on example model easiest strategy we only procedure on limited quantity of insights and get a little example of realities .

\section{SYSTEM OVERVIEW}

The framework engineering of Smart Health Care framework by Online Media Using Data Mining Technique which shows how the information can be gotten to from client and how does measure venture by stem and furthermore shows how framework create drug for client with additionally considering assessment mining by client 


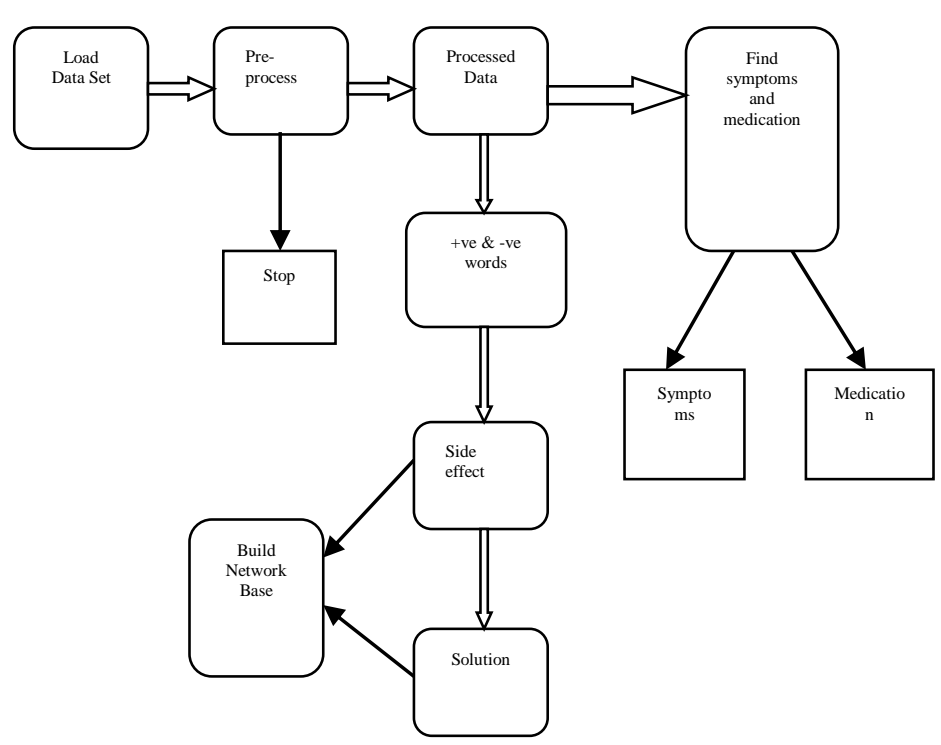

Fig 1. System Architecture

\section{A. Get Dataset}

We are taking information from Disease gathering site. This information is in the type of clients tweets identified with all Disease types and its medicines. Additionally this module gives the office to live tweet and these tweets are taken as an info dataset for handling. These information is identified with medicines and experience of specific medications on a malignant growth so it is a crude information from this we need to discover the how numerous individuals give positive reaction. In the wake of gathering this dataset, information will ship off next cycle for example Text processing.

\section{B. Text Processing}

Text handling is a cycle where we eliminate the stop words also, text stemming

- Stop words expulsion: The words instead of characteristic language words are the stop words. In short stop words are words which are trivial.

- Text stemming: Inflected and inferred words are eliminated in stemming measure. These are eliminating on their stem base or root structure. It for the most part a composed word structures.

\section{Pattern Taxonomy Process}

At the point when the imported archive having enormous size so its become hard to the content processor to measure dataset. So to survive this issue the archive or dataset is break in to the little sections. Presently each section is considered as discrete record. A few terms are separating from positive report and these terms are separating in to each report. Arrangement. Along with this, social and gathering data is more important to cold clients than to hefty clients.

\section{Find out Positive And Negative Words}

Handled information is helpful to discover positive and negative assessment as remarks of client. To discover this we utilized our own predefined word reference in which we add the positive and negative words. To evade the rehashed words we utilized TF-IDF calculation. Term recurrence (TF) check the recurrence of words found in the archive it implies it how often happens a specific word .IDF (Inverse record recurrence) compute the rate of term happens in primary record.

\section{E. Calculate Symptoms and Medication}

By utilizing Our predefined word reference of manifestations words we can discover the side effects from clients tweets. By taking master specialists assessment or utilizing on the web Medical word reference we can rattle off the medicine as per side effects. We can add this data in our information base for best outcome. So that the client can choose their manifestations and came to know medicine for disease so the medical care is improve by utilizing our discussion since its update routinely by clients and administrator. Exploratory outcomes show that multi-see NMF is a best strategy for clinical record grouping. Also, we find that utilizing separated drug/side effect names to bunch clinical archives beats simply utilizing words.

\section{SYSTEM REQUIREMENT}

\section{A. Hardware requirements \\ - Hard disk: $128 \mathrm{~GB}$ \\ - RAM: $512 \mathrm{MB}$ \\ - Processor: Pentium and above \\ - Input device: Keyboard and Mouse \\ - Output device: Monitor}

\section{B. Software requirements \\ - Operating System: Windows 7/Linux \\ - Back End: MySQL, Oracal $10 \mathrm{~g}$ \\ - UML Design: StarUml. PROPOSED METHODOLOGY.}

\section{A. Navies Bayes}

Naive Bayes classifier is a probabilistic machine learning model that's used for classification task. The crux of the classifier is based on the Bayes theorem. Using Bayes theorem, we can find the probability of A happening, given that $\mathrm{B}$ has occurred. Here, $\mathrm{B}$ is the evidence and $\mathrm{A}$ is the hypothesis. The assumption made 


\section{EPRA International Journal of Research and Development (IJRD)}

here is that the predictors/features are independent. That is presence of one particular feature does not affect the other. Hence it is called naive.

\section{B. Support Vector Machine}

$$
\mathrm{P}(\mathrm{A} / \mathrm{B})=\frac{\mathrm{P}(\mathrm{B} / \mathrm{A}) \mathrm{P}(\mathrm{A})}{\mathrm{P}(\mathrm{B})}
$$

Backing Vector Machine (SVM) is an administered machine learning calculation which can be utilized for both order also, relapse difficulties. Be that as it may, it is generally utilized in order issues. In this calculation, we plot every information thing as a point in $\mathrm{n}$ dimensional space (where $\mathrm{n}$ is number of highlights you have) with the estimation of each component being the Worldwide Journal for Advanced innovation and Educational Research in Engineering (IJATERE) estimation of a specific organize. At that point, we perform arrangement zby finding the hyper-plane that separate the two classes very well (take a gander at the underneath depiction).

\section{A. Pattern Taxonomy Process}

$\mathrm{Knn}$ is a non-parametric directed learning strategy in which we attempt to characterize the information highlight a given classification with the assistance of preparing set. In basic words, it catches data of all preparation cases and characterizes new cases in view of a closeness.

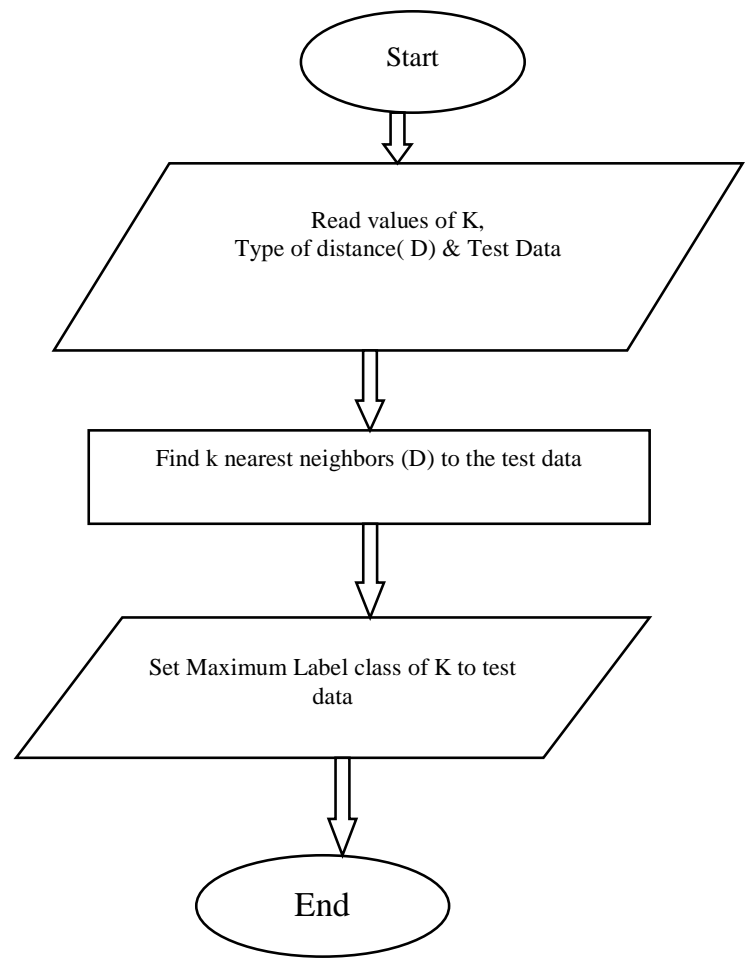

\section{SYSTEM ANALYSIS}

A. TEXT PROCESSING

A numerical model is a portrayal of a framework utilizing numerical ideas and language. The cycle of building up a numerical model is named as numerical

- View patient and specialist data.

- Get assessment related meds.

- Feedback System for Patient.

- Find Symptoms and Medication which is extremely valuable to everybody.

\section{B. SET THEORY}

LET THE SYSTEM BE DESCRIBED BY S,

$\mathrm{S}=\{\mathrm{I}, \mathrm{P}, \mathrm{R}, \mathrm{O}\}$

WHERE, S : IS A SYSTEM.

I: IS INPUT

R: IS SET OF RULES

O: FINAL OUTPUT.

$\mathrm{I}=\{\mathrm{I} 1 ; \mathrm{I} 2 ; \mathrm{I} 3 ; \mathrm{I} 4\}$

WHERE,

$\mathrm{I} 1=$ ENTER PATIENT

I2 = ENTER DOCTOR INFORMATION

$\mathrm{I} 3=$ ENTER DISEASE SYMPTOMS.

I4= FEEDBACK BY PATIENT.

$P$ IS SET OF PROCEDURE OR FUNCTION OR PROCESSES OR METHODS.

$\mathrm{P}=\{\mathrm{P} 1, \mathrm{P} 2, \mathrm{P} 3\}$;

WHERE,

$\mathrm{P} 1=$ CHECK LOGIN FOR PATIENT.

P2 $=$ SEARCHING FOR DOCTOR.

P3 = READ DISEASE SYMPTOM.

P4 = PREDICTING MEDICATION.

P5 $=$ VIEW PATIENT LIST.

R IS SET OF RULES

$\mathrm{R}=\mathrm{R} 1, \mathrm{R} 2$;

$\mathrm{R} 1=$ ENTER VALID INFORMATION.

R2 = MATCH THE DISEASE WITH SYMPTOMS.

$\mathrm{O}=\{\mathrm{O} 1, \mathrm{O} 2, \mathrm{O} 3\}$

WHERE,

O1 = PREDICT MEDICATION.

$\mathrm{O} 2=$ DOWNLOAD PRESCRIPTION PAPER

Fig 2 Working of KNN Algorithm 


\section{VENN DIAGRAM}

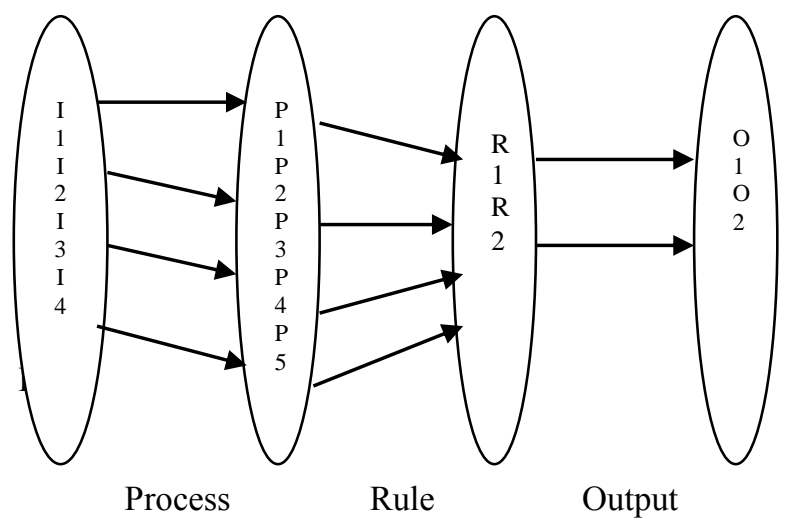

Fig 3: Venn Diagram

Where,

I1, I2, I3. I4 are inputs,

$\mathrm{P} 1, \mathrm{P} 2, \mathrm{P} 3, \mathrm{P} 4, \mathrm{P} 5$ are process

$\mathrm{R} 1 . \mathrm{R} 2$ is rules

And $\mathrm{O} 1, \mathrm{O} 2, \mathrm{O} 3$ are output.

\section{ADVANTAGES OF PROPOSED SYSTEM}

- New disease forum that including all disease related information and data flow .

- Getting knowledge base information related all disease.

- View patient and doctor information.

- Get opinion related medicines.

- Feedback System for Patient.

- Find Symptoms and Medication which is very useful to everyone.

\section{CONCLUSION}

In this Proposed System, we construct a coordinating framework to remove treatment, result manifestation/prescription names from unstructured/semiorganized Data from Disease gathering. The general framework contains persistent treatment, positive Negative impact, Medication name, Symptoms detail, as size of data present $0^{*} \mathrm{n}$ the web has taken a state of the monster it has become a need to expand the productivity of the pursuit Data. Information and information mining on information is very significant on the grounds that we are getting important data which isn't effectively accessible, and all data are continuous data. Our Proposed framework likewise adequately produces drug for each illness and furthermore permits downloading remedy paper.

\section{REFERENCES}

1. J. Huan and J. Prins, Efficient mining of frequent subgraphs in the presence of isomorphism, in Proc. $3 r d$ IEEE Int. Conf. Data Mining, Melbourne, Florida.FL, USA, 2003, pp. 549552.

2. June Almenoff, Joseph M. Tonning, Perspectives on the Use of Data Mining in Pharmacovigilance Leading Article Drug Safety 2005; 28 (11): 981 - 1007 01145916/05/0011-098

3. J. Hans and M. Kamber, Data Mining: Concepts and Techniques. 2nd ed.Burlington, MassMA, USA: Morgan Kaufmann, 2006.

4. D. Hand, Principles of data mining, Drug Safety, vol. 30,pp. 621622, Jul. 2007

5. Ochoa, A. Hernandez, L. Cruz, J. Ponce, F. Montes

6. L. Li, and L. Janacek. Artificial societies and social simulation using ant colony, particle swarm optimization and cultural algorithms, Source: New Achievements in Evolutionary Computation, Book edited by: Peter Korosec, ISBN 978-953-307-053-7, pp. 318, February 2010.

7. Jan Noessner, Mathias Niepert, Christian Meilicke, and Heiner Stucken-schmidt Leveraging Terminological Structure for Object Reconciliation

8. L. Aroyo et al. (Eds.): ESWC 2010, Part II, LNCS 6089, pp. 334348, 2010. c Springer-Verlag Berlin Heidelberg 2010

9. A.Akay and Bjorn-erik Erlandsoon,A novel data mining platform lever-aging social media to monitor outcomes of Januvia. In IEEE EMBS Osaka,Japan,3-7 july,2013.

10. Si Yan and Yanliang Qi, Apply Text Mining to Advance Cancer Research, IJPMBS, Vol. 4, No. 2, April 2015. T.Anisha1 , Mr.N.Thulasi2 Improving Health Care Based on opinion Min-ing Using KNN International Journal of Innovative Research in Science, Engineering and Technology (An ISO 3297: 2007 Certified Organization) Vol. 5, Issue 4, April 2016.

11. S. Arul Kiruba, 1V. Pavithra, 1A. Saranya and $2 B$. Dharani ICON: Improving Care of Human Health using Intelligent Data Mining Advances in Natural and Applied Sciences. 10(2) February 2016

12. Sonali More, P. P. Josh Survey on Social Media Data Mining Techniques IJIRCCE DOI: 10.15680/IJIRCCE.2017. 0504220

13. M.Kiruthika1 , M.Kokilavani2 , N.Mouniga3 , R.Anitha Nithya4 Social Network Based Health Analysis Using User Trust Behavior Model IRJAET E - ISSN: 24544752 P - ISSN : 2454-4744 Vol 3 Issue 1 (2017) Pages 1660 - 1665 Received : 15.02.2017 Published : 26.02.2017 